\title{
Instrument data simulations for GRACE Follow-on: observation and noise models
}

\author{
Neda Darbeheshti ${ }^{1}$, Henry Wegener ${ }^{1}$, Vitali Müller ${ }^{1}$, Majid Naeimi ${ }^{2}$, Gerhard Heinzel ${ }^{1}$, and \\ Martin Hewitson ${ }^{1}$ \\ ${ }^{1}$ Max Planck Institute for Gravitational Physics (Albert Einstein \\ Institute), Leibniz Universität Hannover, Hanover, Germany \\ ${ }^{2}$ Institut für Erdmessung-Leibniz Universität Hannover, Hanover, Germany \\ Correspondence to: Neda Darbeheshti (neda.darbeheshti@aei.mpg.de)
}

Received: 2 June 2017 - Discussion started: 7 August 2017

Revised: 13 October 2017 - Accepted: 16 October 2017 - Published: 17 November 2017

\begin{abstract}
The Gravity Recovery and Climate Experiment (GRACE) mission has yielded data on the Earth's gravity field to monitor temporal changes for more than 15 years. The GRACE twin satellites use microwave ranging with micrometre precision to measure the distance variations between two satellites caused by the Earth's global gravitational field. GRACE Follow-on (GRACE-FO) will be the first satellite mission to use inter-satellite laser interferometry in space. The laser ranging instrument (LRI) will provide two additional measurements compared to the GRACE mission: interferometric inter-satellite ranging with nanometre precision and inter-satellite pointing information. We have designed a set of simulated GRACE-FO data, which include LRI measurements, apart from all other GRACE instrument data needed for the Earth's gravity field recovery. The simulated data files are publicly available via https://doi.org/10.22027/AMDC2 and can be used to derive gravity field solutions like from GRACE data. This paper describes the scientific basis and technical approaches used to simulate the GRACE-FO instrument data.
\end{abstract}

\section{Introduction}

The space gravimetry mission GRACE (Tapley et al., 2004) observes the Earth's gravity field changes with time. GRACE is the first low-low satellite-to-satellite tracking mission: the principal measurement is the distance variability between low-orbit GRACE twin satellites, which translates into the monthly gravity models (Wahr et al., 1998).

Kim (2000) published the first GRACE satellite simulation study before the launch of the GRACE satellites (in 2002). Now, 17 years later, GRACE satellites are at the end of their lifetime and GRACE-FO data will be available soon. Although the GRACE-FO mission and its instrument data streams will be very similar to GRACE, the necessity for a GRACE-FO instrument data simulation emerges from the additional interferometric inter-satellite ranging. Flechtner et al. (2016) have performed a full-scale simulation over the nominal GRACE-FO mission lifetime of 5 years and showed notable improvements with the LRI on a global scale of the order of $23 \%$. Also, the GRACE-FO science data system team at Jet Propulsion Laboratory (JPL) has planned to release a GRACE-FO "Grand Simulation" data set before the real GRACE-FO data are available (Watkins et al., 2016).

Most importantly, the operation of the LRI in addition to the primary K-band ranging (KBR) instrument yields extra information not only in the ranging measurement, but also in the attitude determination. Therefore GRACE-FO LRI data processing will contain precise measurements of the satellites' pitch and yaw angles. In this paper, simulated LRI pitch and yaw angles are provided for the first time. Exploitation of the new GRACE-FO measurements has great potential to improve the spatial and temporal resolution of the Earth's gravity field solutions.

Also, there are different techniques to recover the Earth's gravity field from GRACE-like data (e.g. Reigber, 1989; Gerlach et al., 2003; Mayer-Gürr, 2006; Rummel, 1979). Therefore, simulated instrument data provide a controlled, closed 
form medium to test and improve different gravity field recovery techniques.

We have generated a set of simulated GRACE-FO data for a period of 1 month with a $5 \mathrm{~s}$ sampling rate. The data set is available for download via https://doi.org/10.22027/ AMDC2. The recovered gravity field solutions using this data set can be submitted via the same link. The goals of generating this set of simulated data are

- to improve different gravity field recovery techniques by comparing the input gravity field for the simulation and the recovered gravity fields;

- and to use new LRI data, such as LRI ranging and LRI attitude information, in different gravity field recovery techniques.

The analysis of seasonal or sub-seasonal geophysical features is not the focus of this simulated data set, as the duration of the simulated data is short.

The main purpose of this paper is to describe the chain of instrument data simulation procedures. The first section presents the preliminaries for the data simulation, including the coordinate systems and symbols, followed by each section describing each instrument data simulation with details of the instrument noise models.

\section{Preliminaries}

The following coordinate systems are used to define the various simulated data.

- International Celestial Reference Frame (ICRF) - inertial frame

- origin: centre of mass (CoM) of the Earth

- axes: according to IERS 2010 conventions (Petit and Luzum, 2010)

- International Terrestrial Reference Frame (ITRF) Earth-fixed (co-rotating) frame

- origin: CoM of the Earth

- axes: according to IERS 2010 conventions (Petit and Luzum, 2010)

- Line-of-sight frame (LOSF), one per satellite for GRACE A

- origin: satellite CoM

$-x_{\mathrm{LOSF}_{\mathrm{A}}}=\frac{\boldsymbol{r}_{\mathrm{B}}-\boldsymbol{r}_{\mathrm{A}}}{\left|\boldsymbol{r}_{\mathrm{B}}-\boldsymbol{r}_{\mathrm{A}}\right|}$, where $\boldsymbol{r}$ is the satellites' position vector in the ICRF (i.e. line-of-sight vector and roll axis)

$-\boldsymbol{y}_{\mathrm{LOSF}_{\mathrm{A}}}=\frac{\boldsymbol{x}_{\mathrm{LOSF}_{\mathrm{A}}} \times \boldsymbol{r}_{\mathrm{A}}}{\left|\boldsymbol{x}_{\mathrm{LOSF}_{\mathrm{A}}} \times \boldsymbol{r}_{\mathrm{A}}\right|}$ (i.e. pitch axis)
$-z_{\mathrm{LOSF}_{\mathrm{A}}}=\boldsymbol{x}_{\mathrm{LOSF}_{\mathrm{A}}} \times \boldsymbol{y}_{\mathrm{LOSF}_{\mathrm{A}}}$ (i.e. yaw axis; for GRACE $\mathrm{B}$, the $\mathrm{A}$ and $\mathrm{B}$ indices should be exchanged)

- Satellite frame (SF), one per satellite according to Case et al. (2002)

- origin: satellite CoM

$-x_{\mathrm{SF}}=$ from the origin to a target location of the phase centre of the K- or Ka-band horn

- $\boldsymbol{y}_{\mathrm{SF}}=$ forms a right-handed triad with $\boldsymbol{x}_{\mathrm{SF}}$ and $z_{\mathrm{SF}}$

$-z_{\mathrm{SF}}=$ normal to $x_{\mathrm{SF}}$ and to the plane of the main equipment platform and positive towards the satellite radiator on the bottom of the GRACE-FO

The LOSF and SF are shown in Fig. 1. Since we did not model variations in the satellites' CoM (and the CoM coinciding with the on-board accelerometer's proof masses) for data simulation, the SF coincides with the science reference frame defined in Case et al. (2002).

All simulated data are published in GRACE Level-1B data format: daily files with a $5 \mathrm{~s}$ sampling rate (Case et al., 2002). They can be considered pre-processed like GRACE Level1B data. Time tags are given in GRACE GPS seconds, i.e. seconds since epoch 1 January 2000, 12:00:00 (no leap seconds applied). Five instrument data types were simulated; the following sections in this paper describe each simulated instrument's observations and errors, respectively.

- GPS navigation data (GNV1B)

Simulated GPS positions and velocities are the output of the orbit integrator, which are rotated from ICRF to ITRF, and a GPS error is added to each. The error-free positions can be considered a kinematic orbit.

- K-band ranging system (KBR1B)

Simulated KBR ranging data are derived from the errorfree GPS positions and velocities with added KBR errors.

- Star camera (SCA1B)

Simulated star camera quaternions are derived from the simulated roll, pitch, and yaw angles with added errors.

\section{- Accelerometer (ACC1B)}

Simulated linear accelerations are calculated from the non-gravitational accelerations acting on the satellites. The error-free simulated star camera quaternions are used to transform the linear accelerations from ICRF to SF. Then accelerometer noise, scale, and bias are added. The angular accelerations are calculated from the errorfree simulated star camera quaternions. 

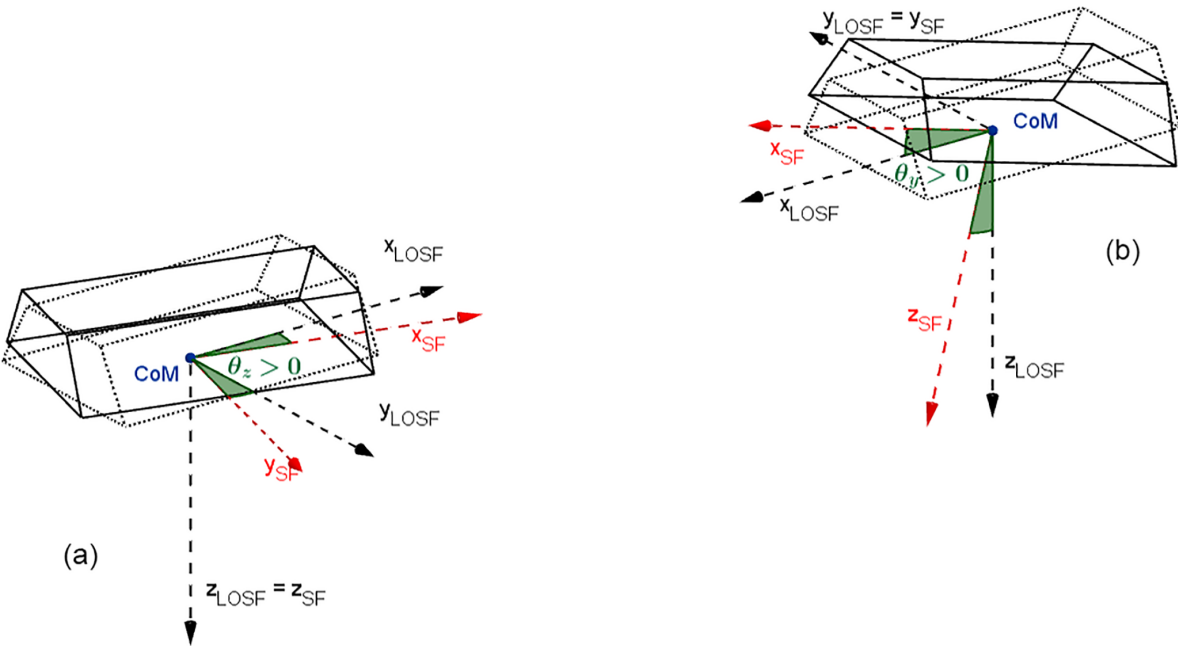

Figure 1. Illustration of SF and LOSF for GRACE satellites. Small positive yaw (left) and pitch (right) angles indicate the direction of rotation defining the sign of the pointing angles.

\section{- Laser ranging instrument (LRI1B)}

Simulated LRI ranging data are derived from error-free GPS positions and velocities with added LRI errors.

Figure 2 shows a flow chart of the procedure used for the simulations. For each instrument, first the error-free observation was generated, and then the errors including instrument noise, bias, and scale were applied to each instrument observation.

In this paper, the symbols $\delta$ and $\Delta$ are used for timevarying and constant errors, respectively. The symbol $\widetilde{\delta}$ denotes amplitude spectral densities (ASDs). For data simulations, a five-point numerical differentiation method was used for the numerical differentiations. The LISA Technology Package Data Analysis (LTPDA) toolbox (https://www. elisascience.org/ltpda/) for MATLAB was used for the generation of time series based on instrument noise models given in terms of ASD. LTPDA uses Franklin's random noise generator method (Franklin, 1965) to generate arbitrarily long time series with a prescribed spectral density.

\section{Simulating GNV1B data}

An orbit integrator is used to calculate the trajectories of the GRACE-FO satellites (GRACE-FO A and GRACE-FO B) through the numerical integration of Newton's second law of motion based on knowledge of the forces acting on the satellite. Table 1 summarises the orbit integrator parameters.

The IERS2010 conventions are used for rotation between the ITRF and the International Celestial Reference Frame (ICRF). Two types of force models were used for orbit integration.

- Gravitational forces:

- A static gravity field of a certain degree and order
Table 1. Orbit integrator parameters.

\begin{tabular}{ll}
\hline Parameter & Description \\
\hline Altitude & $477.7 \mathrm{~km}$ \\
Eccentricity & 0.0019 \\
Inclination & $89.0081^{\circ}$ \\
Numerical integration approach & Gauss-Jackson order 12 \\
Integration length & 31 days (May 2005) \\
Integration step size & $5 \mathrm{~s}$ \\
\hline
\end{tabular}

- The ocean tide model EOT11a (Rieser et al., 2012) up to degree and order 80

- Direct tides of the Moon and Sun using NASA JPL DE405 ephemeris (Standish, 1998)

- Anelastic solid Earth tides according to IERS2010

- Non-gravitational forces:

- Atmospheric drag model

- Solar radiation pressure model

The static gravity model and its exact degree and order are the unknowns for the gravity field recovery. The degree and order that were used as input are between 75 and 95 . The atmospheric drag and solar radiation pressure models are described in Appendix A. Other gravitational forces, such as atmosphere and ocean short-term mass variations, are not used as this simulation data set focuses on the impact of instrument data errors.

The input to the orbit integrator is the initial time and state (position and velocity vectors) of GRACE-FO A and GRACE-FO B at time 00:00:00 on 1 May 2005. It calculates the two trajectories separately in addition to the time series of accelerations along the trajectory from the gravitational 


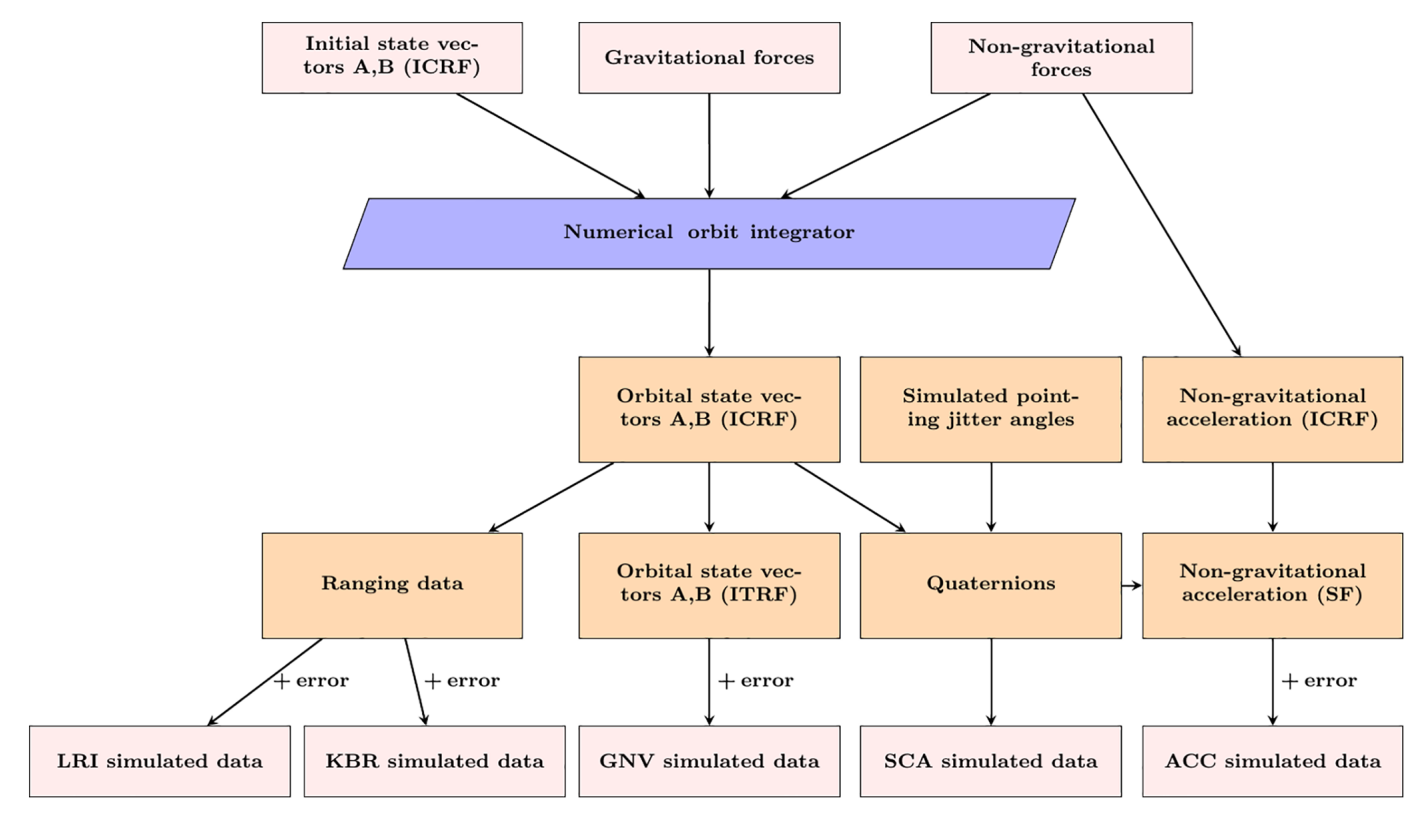

Figure 2. Flow chart of the simulation steps for GRACE-FO instrument data; please refer to Fig. 6 for a detailed description of SCA simulated data.

and non-gravitational force models. The outputs of the orbit integrator are the time series of the position, velocity, and acceleration vectors of GRACE-FO A and GRACE-FO B.

$\boldsymbol{r}_{\mathrm{A}}, \quad \dot{\boldsymbol{r}}_{\mathrm{A}}, \quad \ddot{\boldsymbol{r}}_{\mathrm{A}}, \quad \boldsymbol{r}_{\mathrm{B}}, \quad \dot{\boldsymbol{r}}_{\mathrm{B}}, \quad \ddot{\boldsymbol{r}}_{\mathrm{B}}$

White noise with a level of a few $\mathrm{cm} \mathrm{Hz}^{-1}$ was generated along the $x, y$, and $z$ axes independently and added to each satellite position:

$\boldsymbol{r}_{\mathrm{GNV} 1 \mathrm{~B}}=\boldsymbol{r}+\delta \boldsymbol{r}_{\mathrm{GNV} 1 \mathrm{~B}}$.

Then the noise was differentiated numerically and added to the velocities along the $x, y$, and $z$ axes separately for each satellite:

$\dot{\boldsymbol{r}}_{\mathrm{GNV} 1 \mathrm{~B}}=\dot{\boldsymbol{r}}+\delta \dot{\boldsymbol{r}}_{\mathrm{GNV} 1 \mathrm{~B}}$.

\section{Simulating SCA1B data}

The satellite attitude with respect to the ICRF is determined from the star cameras on-board the satellites. The measured attitude is expressed in terms of quaternions $q$ :

$q=\left(\begin{array}{llll}q_{0} & q_{1} & q_{2} & q_{3}\end{array}\right)$.

Here, $q_{0}$ denotes the real component and $q_{1}, q_{2}$, and $q_{3}$ are the imaginary components of the quaternion. The time series of quaternions is provided in the SCA1B product.

An attitude and orbit control system keeps the satellite orientation near its nominal attitude within a certain boundary for each of the three pointing angles. These boundaries have been lowered for GRACE-FO compared to GRACE for two reasons. The first is due to the coupling of pointing angle errors into the ranging data; experience has shown that improved pointing would enhance the quality of gravity field solutions (Horwath et al., 2011). Secondly, the LRI requires better satellite pointing in order to guarantee its functionality; otherwise there is a risk that the laser beam will start to hit obstacles. Hence, the combined effect of pointing jitter on one hand and frame misalignments on the other hand cannot exceed a certain value (a few milliradians in terms of pitch and yaw angles for GRACE-FO). This yields strict requirements for the construction and mounting of the LRI components and also the necessity for an improved pointing control.

The pointing jitter angles describe how the "true" satellite orientation (as it actually is) deviates from the "nominal" orientation (as it should be ideally in the absence of pointing angles). The nominal orientation is satellites' attitude reference. We assumed that the satellites' attitude reference is the alignment of SF and LOSF for the simulations.

Accordingly, satellite pointing angles can be computed from star camera quaternions and orbital positions (described in Appendix B). For simulating star camera quaternions, one has to go the opposite way. The pointing angles from GRACE-FO attitude and orbital control system performance predictions were provided to us by JPL and AIRBUS Defense and Space. A model based on the spectrum of these predicted angles was used to simulate the pointing angles. The common approach for generating time series with a known spectrum is to use a random noise generator. Figure 3 shows the ASD of the simulated roll $\left(\theta_{x}\right)$, pitch $\left(\theta_{y}\right)$, and yaw $\left(\theta_{z}\right)$ angles. One can see that all three angles have peaks mostly in 


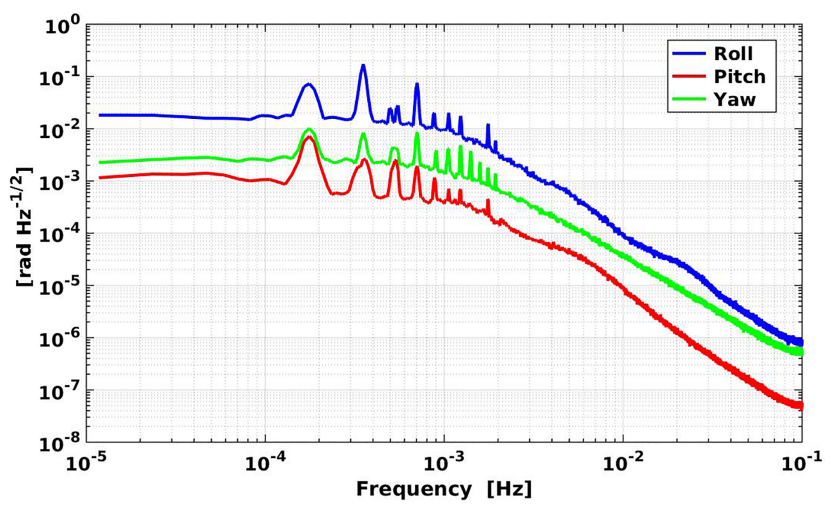

Figure 3. ASD of simulated roll, pitch, and yaw angles.

the frequency band between $10^{-4}$ and $2 \times 10^{-3}$. These peaks disturb the functionality of the random noise generator, and thus they were modelled individually. The result is a time series of error-free inter-satellite pointing angles.

To simulate star camera measurements, white noise $\left(\delta \theta_{\mathrm{SCA} 1 \mathrm{~B}}\right)$ and a bias $\left(\Delta \theta_{\mathrm{SCA} 1 \mathrm{~B}}\right)$ were added to each errorfree angle separately:

$\theta_{x, \mathrm{SCA} 1 \mathrm{~B}}=\theta_{x}+\delta \theta_{x, \mathrm{SCA} 1 \mathrm{~B}}+\Delta \theta_{x, \mathrm{SCA} 1 \mathrm{~B}}$

$\theta_{y}, \mathrm{SCA} 1 \mathrm{~B}=\theta_{y}+\delta \theta_{y, \mathrm{SCA} 1 \mathrm{~B}}+\Delta \theta_{y, \mathrm{SCA} 1 \mathrm{~B}}$

$\theta_{z, \mathrm{SCA} 1 \mathrm{~B}}=\theta_{z}+\delta \theta_{z, \mathrm{SCA} 1 \mathrm{~B}}+\Delta \theta_{z, \mathrm{SCA} 1 \mathrm{~B}}$.

Here, $\theta_{x}, \theta_{y}$, and $\theta_{z}$ are the error-free simulated roll, pitch, and yaw angles; $\theta_{x}, \mathrm{SCA}, \theta_{y}, \mathrm{SCA}$, and $\theta_{z, \mathrm{SCA}}$ are simulated star camera roll, pitch, and yaw angles.

The GRACE-FO satellites are equipped with improved star cameras compared to GRACE, and the number of star camera heads will increase from two to three per satellite (Gath, 2016). Bandikova et al. (2012) suggested that a proper combination of the different star camera heads reduces the high-frequency noise of the pointing angles. Accordingly, it is expected that a better estimation of pointing angles from GRACE-FO star camera data will be available. Therefore, white noise with a level of a few tens of $\mu \mathrm{rad} \mathrm{Hz}^{-1 / 2}$ was chosen, which is lower than the current noise level in roll, pitch, and yaw angles estimated from the GRACE star camera data. The GRACE star cameras are strong in the roll axis and weak in the pitch and yaw axes due to the orientation in which they were mounted (Harvey, 2016). GRACE data (Fig. 4) confirm the $150-300 \mu \mathrm{rad} \mathrm{Hz}^{-1 / 2}$ accuracy for pitch and yaw and $25-35 \mu \mathrm{rad} \mathrm{Hz}^{-1 / 2}$ for roll, which meet the mission requirements (Stanton et al., 1998).

The value of bias for each angle was chosen in the range of a few milliradians. This level of bias has been investigated by Horwath et al. (2011) based on GRACE Level-1B data. Figure 5 shows simulated star camera roll, pitch, and yaw angles, which are similar to the GRACE inter-satellite pointing variations plot in Bandikova et al. (2012).

From the contaminated simulated pointing angles of Eq. (4), the rotation matrix $\mathbf{R}_{\mathrm{SF}}^{\mathrm{LOSF}}$ was built, and with

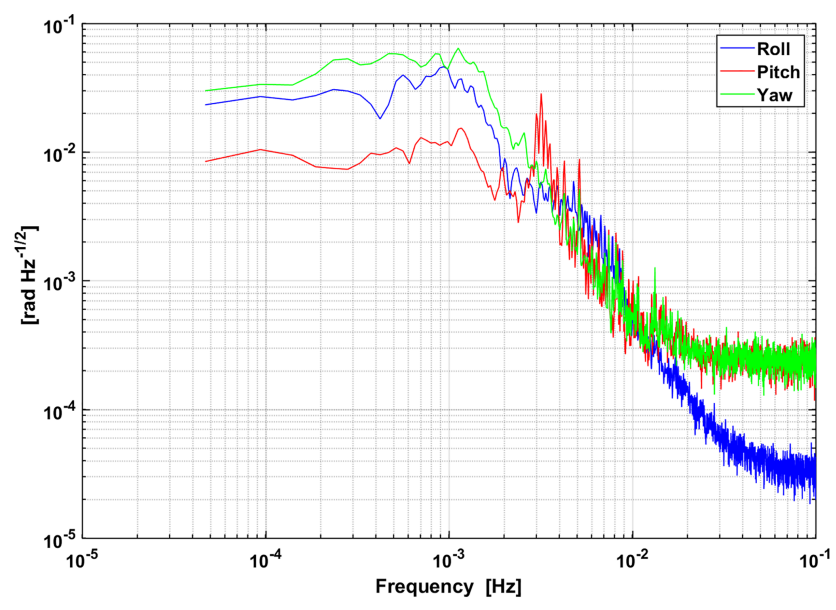

Figure 4. ASD of GRACE roll, pitch, and yaw angles on 1 December 2008 .
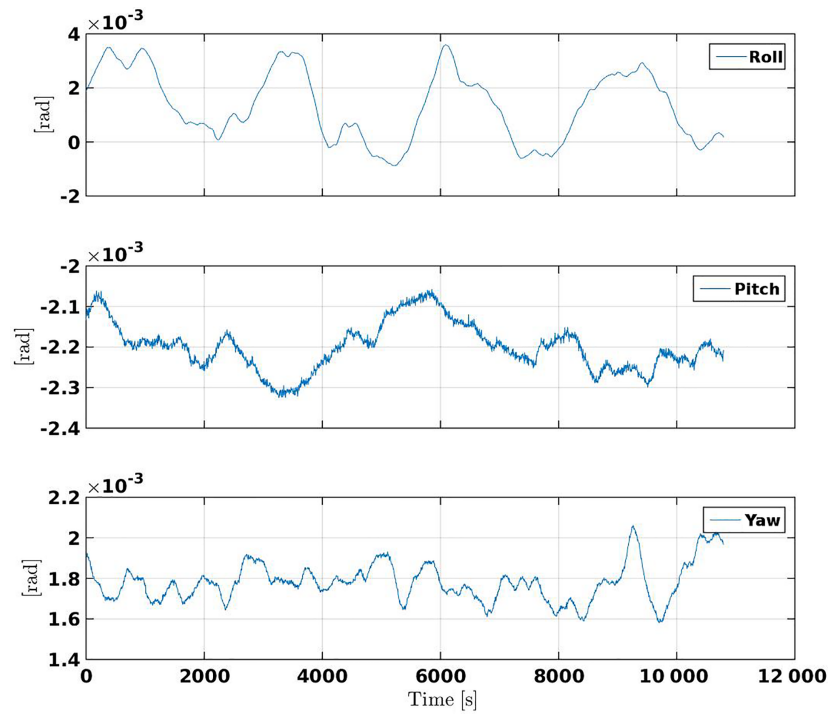

Figure 5. Simulated star camera roll, pitch, and yaw angles during two orbital revolutions for GRACE-FO A.

the error-free simulated orbit positions, the rotation matrix $\mathbf{R}_{\mathrm{LOSF}}^{\mathrm{ICRF}}$ was built. With these two matrices, the matrix $\mathbf{R}_{\mathrm{SF}}^{\mathrm{ICR}}$ is

$\mathbf{R}_{\mathrm{SF}}^{\mathrm{ICRF}}=\mathbf{R}_{\mathrm{LOSF}}^{\mathrm{ICRF}} \cdot \mathbf{R}_{\mathrm{SF}}^{\mathrm{LOSF}}$,

containing the simulated star camera quaternions (Fig. 6). Finally, the simulated quaternions can be recovered from the rotation matrix $\mathbf{R}_{\mathrm{SF}}^{\mathrm{ICRF}}$ by using the equation series (Wu et al., 


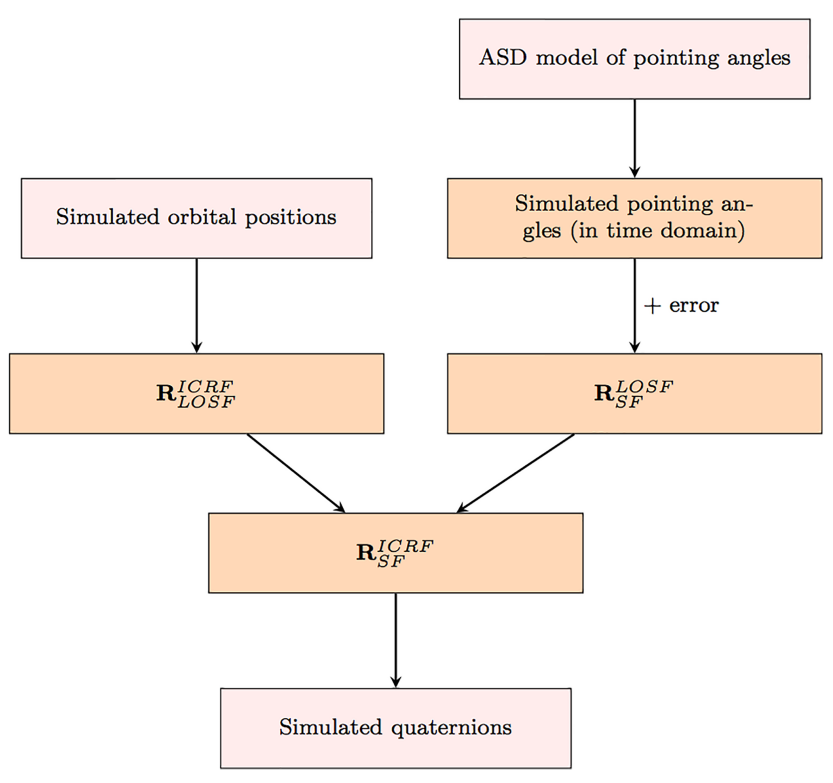

Figure 6. Flow chart of the simulation steps for SCA1B data.

2006):

$q_{0}=\frac{1}{2} \sqrt{1+R_{11}+R_{22}+R_{33}}$

$q_{1}=\frac{\left(R_{23}-R_{32}\right)}{4 q_{0}}$

$q_{2}=\frac{\left(R_{31}-R_{13}\right)}{4 q_{0}}$

$q_{3}=\frac{\left(R_{12}-R_{21}\right)}{4 q_{0}}$,

where $R_{i j}$ represents the elements of $\mathbf{R}_{\mathrm{SF}}^{\mathrm{ICRF}}$. Note that the series in Eq. (6) is only numerically stable as long as the trace of $\mathbf{R}$ is non-negative (i.e. not close to -1 ). A numerically stable pseudocode that was used is shown in Appendix C.

Two other sets of quaternions were generated: error-free quaternions from error-free pointing angles in Eqs. (4) and noisy quaternions that come from white-noise-contaminated pointing angles without the bias. We will refer to these two set of quaternions in the following sections.

\section{Simulating ACC1B data}

Figure 2 shows that the non-gravitational accelerations were computed along the orbit in ICRF.

\subsection{Linear accelerations}

The non-gravitational accelerations are the sum of atmospheric drag and solar radiation pressure accelerations (Appendix A) along the orbit in inertial frame (ICRF). The nongravitational accelerations $\ddot{\boldsymbol{r}}^{\mathrm{ICRF}}$ were transformed into the satellite frame $\ddot{r}^{\mathrm{SF}}$ using the rotation matrix $\mathbf{R}_{\mathrm{ICRF}}^{\mathrm{SF}}$ from error-free simulated quaternions:

$\ddot{\boldsymbol{r}}^{\mathrm{SF}}=\mathbf{R}_{\mathrm{ICRF}}^{\mathrm{SF}} \cdot \ddot{\boldsymbol{r}}^{\mathrm{ICRF}}$

After being transformed into the SF, the linear accelerations were multiplied by the scale factors $s_{x}, s_{x}$, and $s_{z}$, and then the accelerometer noise time series $\left(\delta \ddot{\boldsymbol{r}}_{\mathrm{ACC} 1 \mathrm{~B}}\right)$ and the biases $\left(\Delta \ddot{\boldsymbol{r}}_{\mathrm{ACC} 1 \mathrm{~B}}\right)$ were added along the $x, y$, and $z$ axes independently:

$\ddot{\boldsymbol{r}}_{\mathrm{ACC} 1 \mathrm{~B}}^{\mathrm{SF}}=\left[\begin{array}{ccc}s_{x} & 0 & 0 \\ 0 & s_{y} & 0 \\ 0 & 0 & s_{z}\end{array}\right] \cdot \ddot{\boldsymbol{r}}^{\mathrm{SF}}+\delta \ddot{\boldsymbol{r}}_{\mathrm{ACC} 1 \mathrm{~B}}+\Delta \ddot{\boldsymbol{r}}_{\mathrm{ACC} 1 \mathrm{~B}}$.

The ASD noise model of Kim (2000) was used to generate accelerometer noise $\left(\delta \ddot{\boldsymbol{r}}_{\mathrm{ACC} 1 \mathrm{~B}}\right)$ :

$$
\begin{aligned}
& \tilde{\delta} \ddot{\boldsymbol{r}}_{x / z, \mathrm{ACClB}}(f)=10^{-10} \cdot \sqrt{1+\frac{0.005 \mathrm{~Hz}}{f}} \mathrm{~m} \mathrm{~s}^{-2} \mathrm{~Hz}^{-1 / 2} \\
& 10^{-5} \leq f \leq 10^{-1} \\
& \tilde{\delta} \ddot{\boldsymbol{r}}_{y, \mathrm{ACC} 1 \mathrm{~B}}(f)=10^{-9} \cdot \sqrt{1+\frac{0.1 \mathrm{~Hz}}{f}} \mathrm{~m} \mathrm{~s}^{-2} \mathrm{~Hz}^{-1 / 2} \\
& 10^{-5} \leq f \leq 10^{-1}
\end{aligned} .
$$

The $y$ axis in $\mathrm{SF}\left(\boldsymbol{y}_{\mathrm{SF}}\right.$ in Fig. 1) is considered the leastsensitive axis for accelerometer measurements (Kim, 2000). The noise ASD of the sensitive axes and the less-sensitive axis are shown in Fig. 7. A 1-month time series of the accelerometer noise was generated separately for the $x, y$, and $z$ axes from the ASD models and added to the accelerations (Eq. 8). Values close to the GRACE accelerometer scale and bias along each axis were chosen and kept constant for 1 month of the simulated data. Therefore, in total for both satellites, six accelerometer scale parameters and six accelerometer bias parameters should be estimated during the gravity field recovery using 1 month of the simulated data. The scale and bias parameters will be available via https://doi.org/10.22027/AMDC2 for comparison with the estimated ones.

\subsection{Angular accelerations}

The error-free simulated quaternions were used to generate angular accelerations based on the relations between the quaternions and angular accelerations $\left(\dot{\omega}_{x}, \dot{\omega}_{y}, \dot{\omega}_{z}\right)$ (Müller, 2010):

$$
\left[\begin{array}{c}
\dot{\omega}_{x} \\
\dot{\omega}_{y} \\
\dot{\omega}_{z} \\
-2 \Sigma \dot{q}_{m}^{2}
\end{array}\right]=2 \cdot\left[\begin{array}{cccc}
-q_{1} & q_{0} & q_{3} & -q_{2} \\
-q_{2} & -q_{3} & q_{0} & q_{1} \\
-q_{3} & q_{2} & -q_{1} & q_{0} \\
q_{0} & q_{1} & q_{2} & q_{3}
\end{array}\right] \cdot\left[\begin{array}{c}
\ddot{q}_{0} \\
\ddot{q}_{1} \\
\ddot{q}_{2} \\
\ddot{q}_{3}
\end{array}\right],
$$

where $\ddot{q}_{m}$ are the numerically differentiated simulated quaternions. 


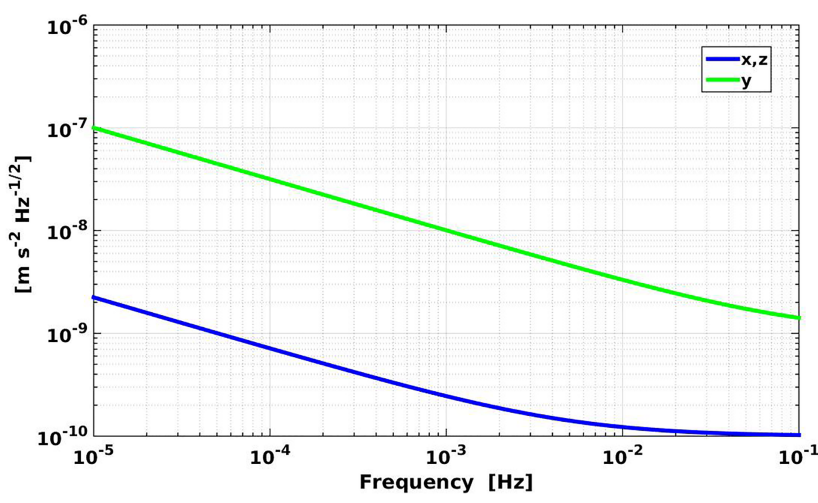

Figure 7. ASD of accelerometer noise.
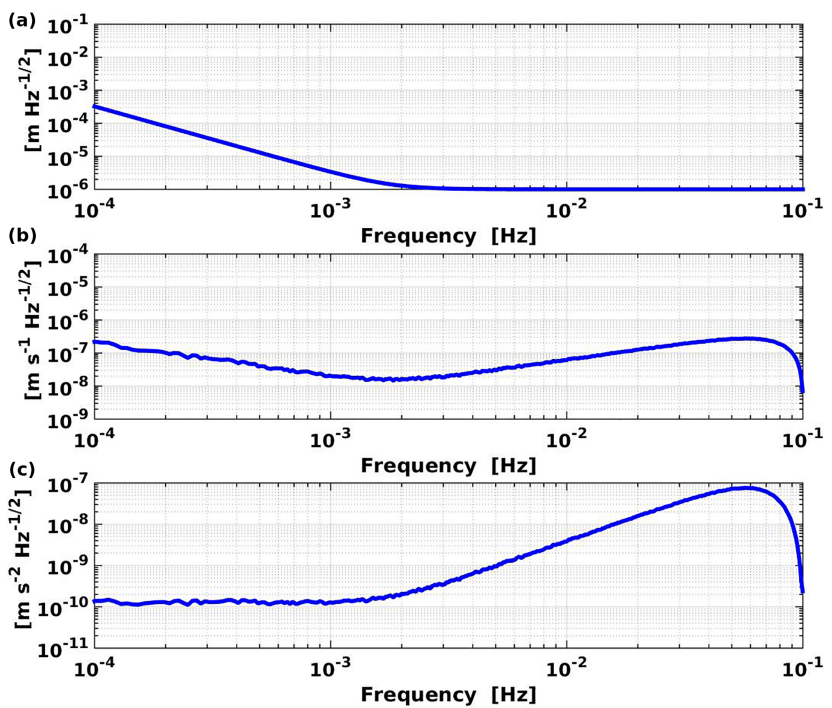

Figure 8. ASD of KBR system and oscillator noise for range (a), range rate (b), and range acceleration (c).

\section{Simulating KBR1B data}

The position, velocity, and acceleration differences of GRACE-FO A and GRACE-FO B are calculated as follows:

$\boldsymbol{r}_{A B}=\boldsymbol{r}_{\mathrm{B}}-\boldsymbol{r}_{\mathrm{A}}$

$\dot{\boldsymbol{r}}_{A B}=\dot{\boldsymbol{r}}_{\mathrm{B}}-\dot{\boldsymbol{r}}_{\mathrm{A}}$

$\ddot{\boldsymbol{r}}_{A B}=\ddot{\boldsymbol{r}}_{\mathrm{B}}-\ddot{\boldsymbol{r}}_{\mathrm{A}}$,

in order to calculate simulated error-free range, range rate, and range acceleration according to

$\rho=\sqrt{\boldsymbol{r}_{\mathrm{AB}} \cdot \boldsymbol{r}_{\mathrm{AB}}}$,

$\dot{\rho}=\frac{\boldsymbol{r}_{\mathrm{AB}}}{\rho} \cdot \dot{\boldsymbol{r}}_{\mathrm{AB}}$,

$\ddot{\rho}=-\frac{\dot{\rho}^{2}}{\rho}+\frac{\dot{\boldsymbol{r}}_{\mathrm{AB}} \cdot \dot{\boldsymbol{r}}_{\mathrm{AB}}}{\rho}+\frac{\boldsymbol{r}_{\mathrm{AB}}}{\rho} \cdot \ddot{\boldsymbol{r}}_{\mathrm{AB}}$.

where $\cdot$ is the vector dot product.
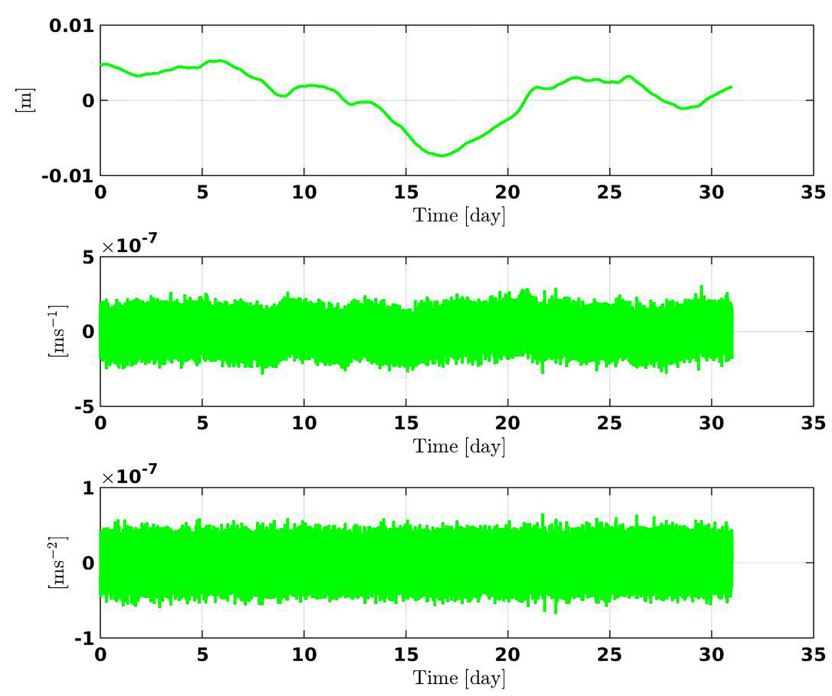

Figure 9. Time series of KBR oscillator and system noise for range (a), range rate (b), and range acceleration (c).

The GRACE-FO KBR instrument (as in GRACE) will measure the biased range between the twin satellites; a bias $(\Delta \rho)$ of a few centimetres was added to the error-free range $(\rho)$. The KBR instrument noise is dominated by system and oscillator noise $\left(\delta \rho_{\mathrm{SO}}\right)$. It was added to the error-free ranging products, as was a geometric error, which is a pointing jitter coupling effect caused by an offset of the KBR antenna phase centre for each satellite $\mathrm{A}$ and $\mathrm{B}\left(\delta \rho_{\mathrm{APC}}\right)$ :

$$
\begin{aligned}
& \rho_{\mathrm{KBR} 1 \mathrm{~B}}=\rho+\delta \rho_{\mathrm{SO}}+\delta \rho_{\mathrm{APC}_{\mathrm{A}}}+\delta \rho_{\mathrm{APC}_{\mathrm{B}}}+\Delta \rho_{\mathrm{KBR} 1 \mathrm{~B}} \\
& \dot{\rho}_{\mathrm{KBR} 1 \mathrm{~B}}=\dot{\rho}+\delta \dot{\rho}_{\mathrm{SO}}+\delta \dot{\rho}_{\mathrm{APC}_{\mathrm{A}}}+\delta \dot{\rho}_{\mathrm{APC}_{\mathrm{B}}} \\
& \ddot{\rho}_{\mathrm{KBR} 1 \mathrm{~B}}=\ddot{\rho}+\delta \ddot{\rho}_{\mathrm{SO}}+\delta \ddot{\rho}_{\mathrm{APC}_{\mathrm{A}}}+\delta \ddot{\rho}_{\mathrm{APC}_{\mathrm{B}}} .
\end{aligned}
$$

In the following, these two error sources are described.

\subsection{System and oscillator noise}

The following ASD model was used to generate KBR noise:

$\tilde{\delta} \rho_{\mathrm{SO}}(f)=10^{-6} \cdot \sqrt{1+\left(\frac{0.0018 \mathrm{~Hz}}{f}\right)^{4}} \mathrm{mHz}^{-1 / 2}$

$10^{-5} \leq f \leq 10^{-1}$.

This ASD model is in agreement with the system and oscillator KBR noise for the satellite pair separation of $238 \mathrm{~km}$ in Kim (2000). Figure 8 illustrates the ASD model. Based on this model, a 1-month time series of the range noise was generated. Then numerical differentiation was used to generate range rate noise and range acceleration noise from the range noise time series (Fig. 9).

\subsection{Antenna phase centre pointing jitter coupling}

The KBR instrument measures the distance between the antenna phase centres, which are placed nominally on the SF 


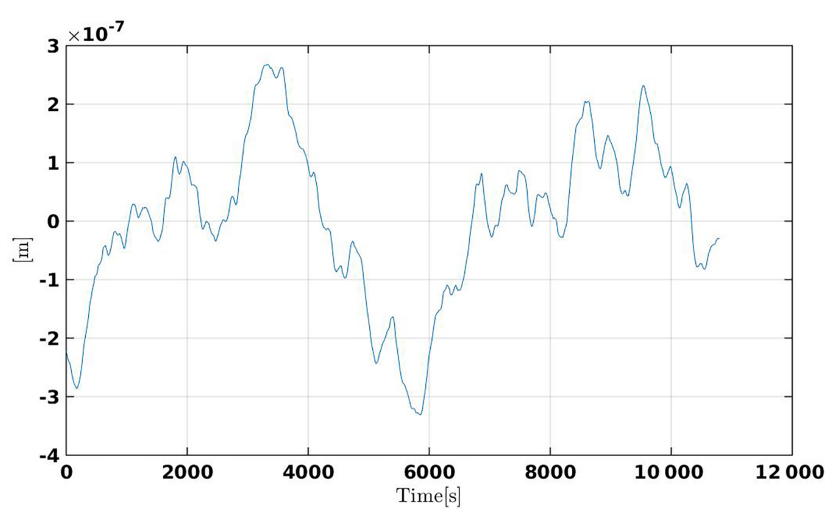

Figure 10. Time series of APC offset pointing jitter coupling with subtracted mean value during two orbital revolutions for GRACEFO A.

$x$ axis almost $1.5 \mathrm{~m}$ away from the satellites' CoM. However, due to manufacturing imperfections and due to the large acceleration of the system during launch, the actual positions differ from the nominal ones. Consequently, any pointing jitter (deviations of the satellites' attitudes from their nominal attitudes) causes a geometric error in the ranging measurement. In the absence of such misplacements and in the absence of pointing jitter, this effect would be zero (rather, constant) and hence not effect the measured (biased) range. Given the antenna phase centre (APC) offset vector $\left(\boldsymbol{p}_{\mathrm{A}}^{\mathrm{SF}}\right)$ in SF and the matrix rotating from SF to ICRF, this error is computed as:

$\delta \rho_{\mathrm{APC}}=-\left(\boldsymbol{e}_{\mathrm{AB}}^{\mathrm{ICRF}}\right)^{T} \cdot \mathbf{R}_{\mathrm{SF}}^{\mathrm{ICRF}} \cdot \boldsymbol{p}_{\mathrm{A}}^{\mathrm{SF}}$

i.e. it is the APC offset (w.r.t. CoM) projected onto the line of sight. For the simulation, the $\mathbf{R}_{\mathrm{SF}}^{\mathrm{ICRF}}$ was calculated from Eq. (B1) using the error-free simulated quaternions, and the line-of-sight vector $\left(\boldsymbol{e}_{\mathrm{AB}}^{\mathrm{ICRF}}\right)$ was calculated from the errorfree satellite positions in ICRF:

$\boldsymbol{e}_{\mathrm{AB}}^{\mathrm{ICRF}}=\frac{\boldsymbol{r}_{\mathrm{B}}-\boldsymbol{r}_{\mathrm{A}}}{\left|\boldsymbol{r}_{\mathrm{B}}-\boldsymbol{r}_{\mathrm{A}}\right|}$

For GRACE-FO B, the indices A and B should be swapped in Eqs. (16) and (17). Figure 10 shows time series of the APC offset pointing jitter coupling for 1 month of GRACE-FO A.

In GRACE, there have been calibration manoeuvres in order to try and estimate the APC offset vectors $\left(\boldsymbol{p}_{\mathrm{A}}^{\mathrm{SF}}, \boldsymbol{p}_{\mathrm{B}}^{\mathrm{SF}}\right)$. The estimates have been published by JPL in the VKB1B files (Case et al., 2002). For the simulations, values of similar magnitude were chosen. These values are not directly given to the user; however, the simulated KBR1B files include a column of simulated estimated correction terms. This means that it is computed from the imperfect attitude information that is provided via simulated SCA1B files. Real GRACE KBR1B data also contain this column, which is called the antenna offset correction (AOC) term (Case et al., 2002). It has to be added to the KBR ranging measurement, so it describes the negative of the error term:

$\mathrm{AOC}_{\rho} \approx-\delta \rho_{\mathrm{APC}_{\mathrm{A}}}-\delta \rho_{\mathrm{APC}_{\mathrm{B}}}$

For the simulations, the correction term of $\mathrm{AOC}_{\rho}$ was computed according to Eq. (16), with the difference that the matrix $\mathbf{R}_{\mathrm{SF}}^{\mathrm{ICRF}}$ was derived from the simulated noisy quaternions without the bias.

A second and third column are also provided that are computed by using numerical differentiation and describe the correction for range rate and range acceleration:

$\mathrm{AOC}_{\dot{\rho}} \approx-\delta \dot{\rho}_{\mathrm{APC}_{\mathrm{A}}}-\delta \dot{\rho}_{\mathrm{APC}_{\mathrm{B}}}$
$\mathrm{AOC}_{\ddot{\rho}} \approx-\delta \ddot{\rho}_{\mathrm{APC}_{\mathrm{A}}}-\delta \ddot{\rho}_{\mathrm{APC}_{\mathrm{B}}}$.

\section{Simulating LRI1B data}

The structure of the LRI1B data file is similar to the KBR1B file, but it contains two additional observations of pitch and yaw angles. Tables D1 and D2 in Appendix D show the format of the data records for simulated KBR1B and LRI1B files. The simulated error-free range, range rate, and range accelerations are calculated from Eqs. (11), (12), and (13). Apart from a bias of a few centimetres, various other errors were added:

$$
\begin{aligned}
\rho_{\mathrm{LRI} 1 \mathrm{~B}} & =\alpha \cdot\left(\rho+\delta \rho_{\mathrm{LF}}+\delta \rho_{\mathrm{TMA}_{\mathrm{A}}}+\delta \rho_{\mathrm{TMA}_{\mathrm{B}}}\right. \\
& \left.+\delta \rho_{\mathrm{ALQ}_{\mathrm{A}}}+\delta \rho_{\mathrm{ALQ}_{\mathrm{B}}}+\Delta \rho_{\mathrm{LRI} 1 \mathrm{~B}}\right) \\
\dot{\rho}_{\mathrm{LRI} 1 \mathrm{~B}} & =\alpha \cdot\left(\dot{\rho}+\delta \dot{\rho}_{\mathrm{LF}}+\delta \dot{\rho}_{\mathrm{TMA}_{\mathrm{A}}}+\delta \dot{\rho}_{\mathrm{TMA}_{\mathrm{B}}}\right. \\
& \left.+\delta \dot{\rho}_{\mathrm{ALQ}_{\mathrm{A}}}+\delta \dot{\rho}_{\mathrm{ALQ}_{\mathrm{B}}}\right) \\
\ddot{\rho}_{\mathrm{LRI} 1 \mathrm{~B}} & =\alpha \cdot\left(\ddot{\rho}+\delta \ddot{\rho}_{\mathrm{LF}}+\delta \ddot{\rho}_{\mathrm{TMA}_{\mathrm{A}}}+\delta \ddot{\rho}_{\mathrm{TMA}_{\mathrm{B}}}\right. \\
& \left.+\delta \ddot{\rho}_{\mathrm{ALQ}_{\mathrm{A}}}+\delta \ddot{\rho}_{\mathrm{ALQ}_{\mathrm{B}}}\right) .
\end{aligned}
$$

In Eq. (20), $\alpha=1+10^{-6}$ is a scale factor which is due to the limited accuracy of the absolute laser frequency value for the phase to length conversion. The three main LRI noise sources in Eq. (20) are laser frequency (LF) noise $\left(\delta \rho_{\mathrm{LF}}\right)$, the coupling of the pointing jitter into the length measurement via triple mirror assembly (TMA; Fig. 11) for each satellite $\mathrm{A}$ and $\mathrm{B}\left(\delta \rho_{\mathrm{TMA}}\right)$, and the additional linear and quadratic pointing jitter coupling ( $\left.\delta \rho_{\mathrm{ALQ}}\right)$. This is a selection of relatively well-known LRI error sources in which LF and TMA errors are expected to be the dominating ones. For the range rate and range acceleration noise, the errors were numerically differentiated. In the following, the LRI error sources are described in detail.

\subsection{Laser frequency noise}

Based on LRI cavity performance tests carried out by JPL, the current best estimate of the ASD of the laser frequency 


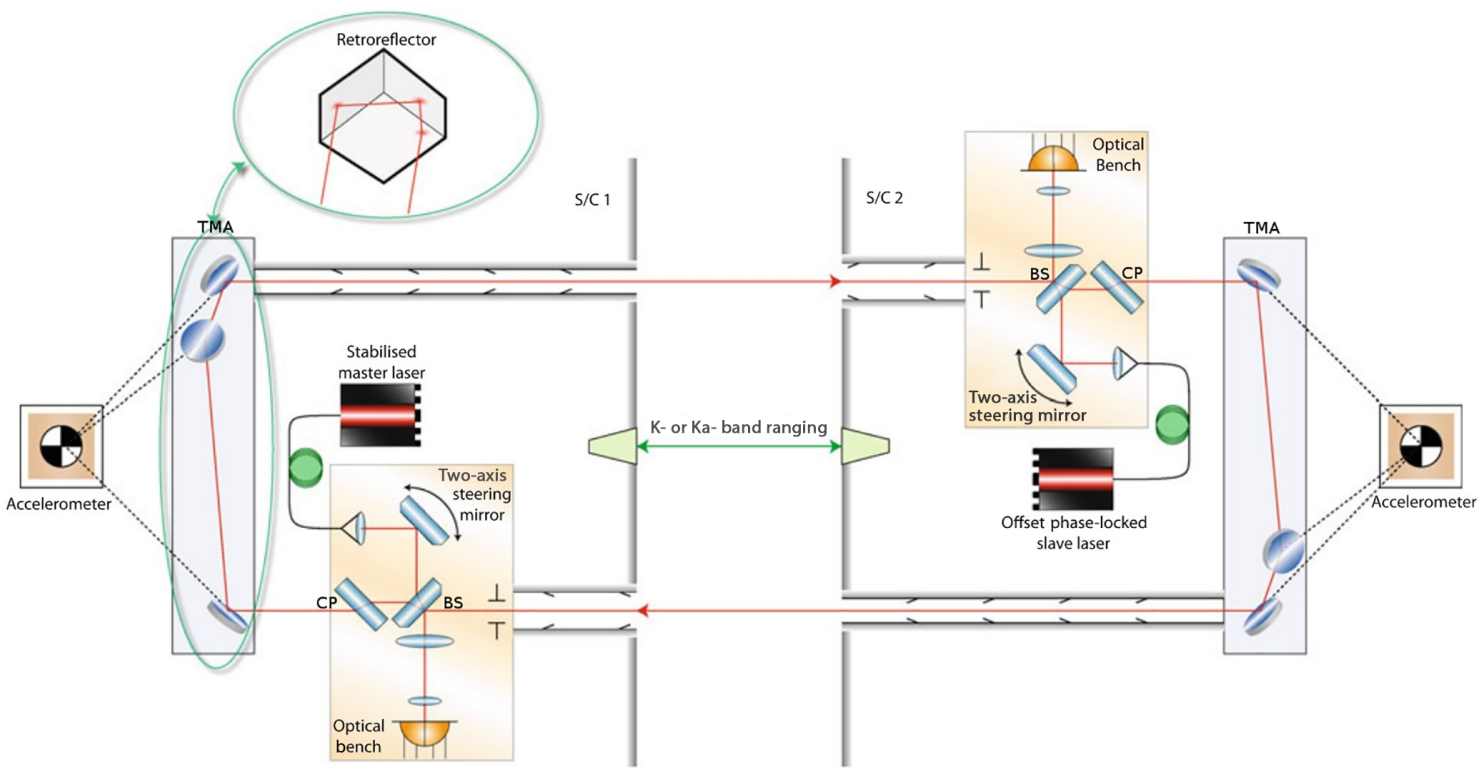

Figure 11. GRACE-FO laser ranging instrument optical layout (from Sheard et al., 2012). BS is beam splitter, CP is compensation plate, and TMA is triple mirror assembly.
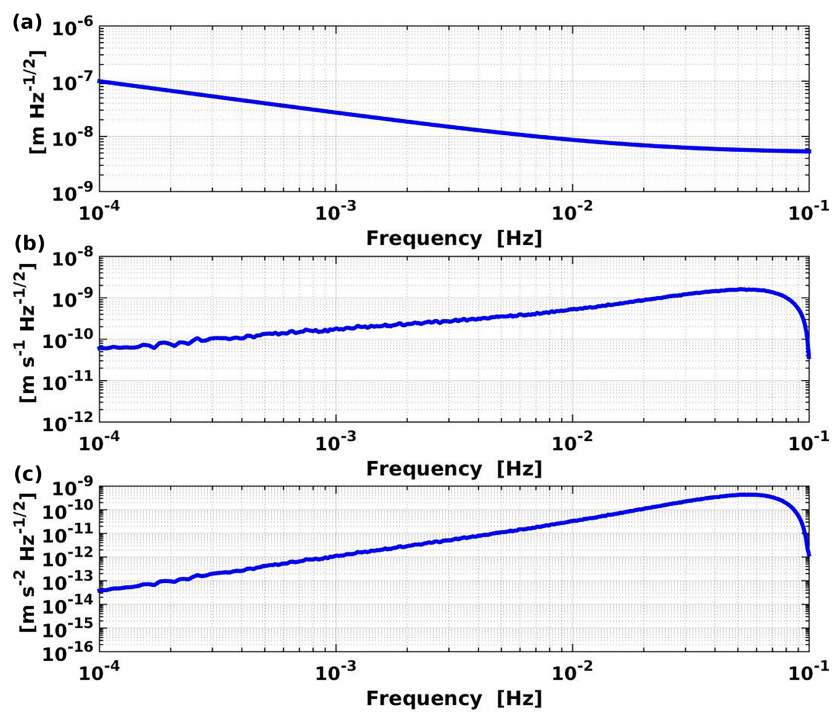

Figure 12. ASD of laser frequency noise for range (a), range rate (b), and range acceleration (c).

noise (i.e. the ranging noise which is induced by the frequency jitter of the LRI master laser) for a satellite separation of $238 \mathrm{~km}$ is

$\tilde{\delta} \rho_{\mathrm{LF}}(f)=5 \times 10^{-9} \cdot \sqrt{1+\left(\frac{0.0182 \mathrm{~Hz}}{f}\right)^{2}} \mathrm{mHz}^{-1 / 2}$.

Figure 12 illustrates this noise. Note that this specific ASD corresponds to a constant satellite separation (of $238 \mathrm{~km}$ ), which is a sufficient simplification for the purpose of generating noise time series.
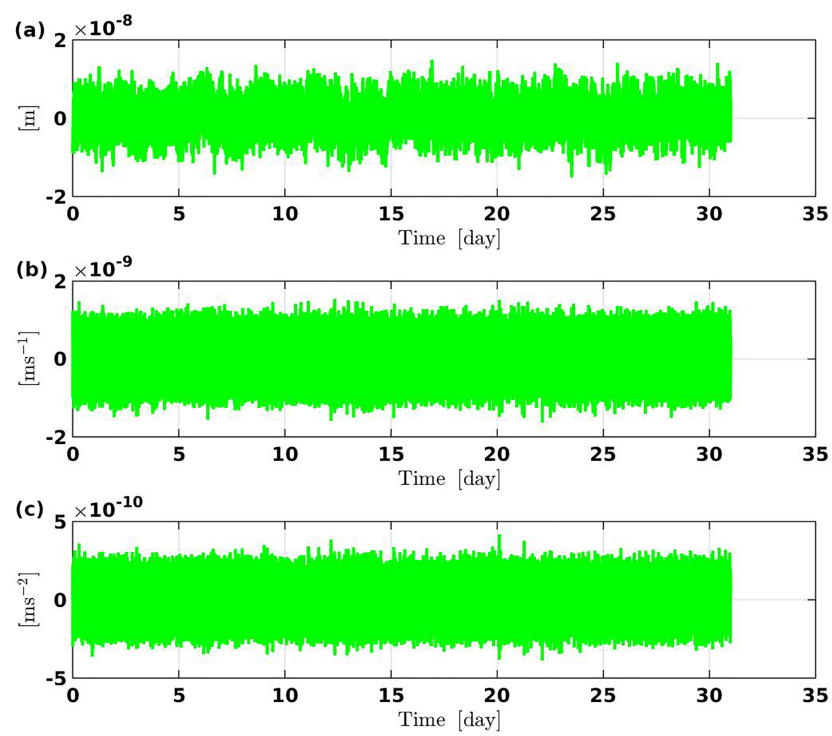

Figure 13. Time series of laser frequency noise for range (a), range rate (b), and range acceleration (c).

A 1-month time series of the range noise $\delta \rho_{\mathrm{LF}}$ was generated from the ASD model (Fig. 12). Then numerical differentiation was used to generate range rate noise and range acceleration noise from the noise range time series (Fig. 13).

\subsection{Triple mirror assembly pointing jitter coupling}

With a good approximation, the LRI measures the biased distance between the TMA vertices of the twin satellites (Fig. 11). Both the pointing jitter and frame misalignments 


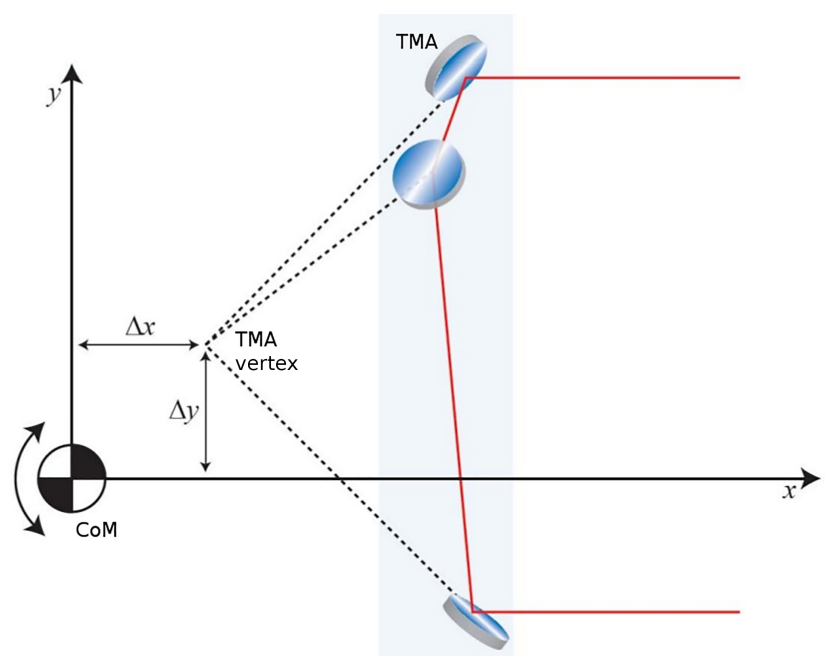

Figure 14. Triple mirror assembly vertex offset from the satellites' centre of mass in two dimensions.

couple into the LRI ranging measurement. This effect is in principle the same as the geometric error effect due to the APC position in the KBR measurement. The only difference is that the nominal positions of the TMA vertices are in the $\mathrm{CoM}$, whereas the nominal positions of the APC are almost $1.5 \mathrm{~m}$ in the SF $x$ direction away from this point.

An offset of the TMA vertex from the satellites' CoM leads to the coupling of satellite pointing jitter into the round-trip length variations measured by the LRI (Fig. 14). The magnitudes of TMA vertex offset vectors $\left(\boldsymbol{v}^{\mathrm{SF}}\right)$ along the $x, y$, and $z$ axes were chosen in the order of a few hundred micrometres. The real values after the GRACE-FO launch are unknown and will have to be calibrated. To calculate $\delta \rho_{\text {TMA }}$, the TMA vertex offset vector $\left(v^{\mathrm{SF}}\right)$ is rotated from the SF into the ICRF and then projected onto the line of sight:

$\delta \rho_{\mathrm{TMA}_{\mathrm{A}}}=-\left(\boldsymbol{e}_{\mathrm{AB}}^{\mathrm{ICRF}}\right)^{T} \cdot \mathbf{R}_{\mathrm{SF}}^{\mathrm{ICRF}} \cdot \boldsymbol{v}_{\mathrm{A}}^{\mathrm{SF}}$,

where $\boldsymbol{e}_{\mathrm{AB}}^{\mathrm{ICRF}}$ is the line-of-sight vector in ICRF. Again, for GRACE-FO B, indices A and B should be swapped. Figure 15 shows time series of TMA pointing jitter coupling for 1 month of GRACE-FO A.

Similar to the KBR1B files, LRI1B files contain correction terms - vertex point correction (VPC) terms - for range, range rate, and range acceleration, which were calculated using the simulated noisy quaternions without the bias:

$\begin{aligned} & \mathrm{VPC}_{\rho} \approx-\delta \rho_{\mathrm{TMA}_{\mathrm{A}}}-\delta \rho_{\mathrm{TMA}_{\mathrm{B}}} \\ & \mathrm{VPC}_{\dot{\rho}} \approx-\delta \dot{\rho}_{\mathrm{TMA}_{\mathrm{A}}}-\delta \dot{\rho}_{\mathrm{TMA}_{\mathrm{B}}} \\ & \mathrm{VPC}_{\ddot{\rho}} \approx-\delta \ddot{\rho}_{\mathrm{TMA}_{\mathrm{A}}}-\delta \ddot{\rho}_{\mathrm{TMA}_{\mathrm{B}}} .\end{aligned}$

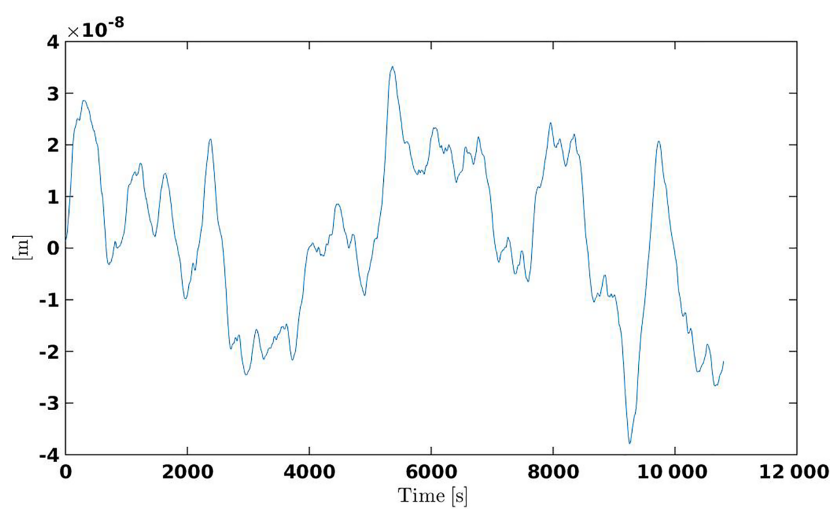

Figure 15. Time series of TMA pointing jitter coupling with subtracted mean value during two orbital revolutions for GRACEFO A.

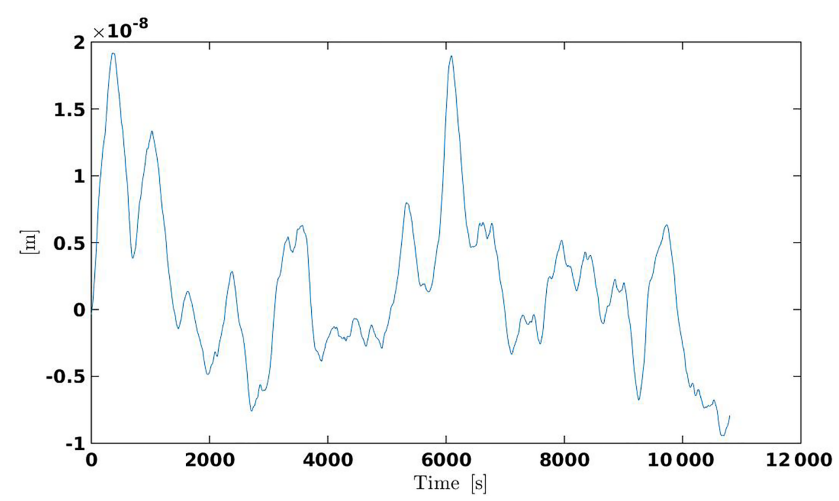

Figure 16. Time series of ALQ pointing jitter coupling with subtracted mean value during two orbital revolutions for GRACEFO A.

\subsection{Additional linear and quadratic pointing jitter coupling}

There is additional linear and quadratic coupling (ALQ) of the pointing jitter angles $\left(\theta_{x}, \theta_{y}\right.$, and $\left.\theta_{z}\right)$ into the length measurements, which can be described as

$$
\begin{aligned}
\delta \rho_{\mathrm{ALQ}} & =\left[c_{x_{\mathrm{A}}} c_{y_{\mathrm{A}}} c_{z_{\mathrm{A}}}\right] \cdot\left[\begin{array}{c}
\theta_{x_{\mathrm{A}}} \\
\theta_{y_{\mathrm{A}}} \\
\theta_{z_{\mathrm{A}}}
\end{array}\right]+\left[\theta_{x_{\mathrm{A}}} \theta_{y_{\mathrm{A}}} \theta_{z_{\mathrm{A}}}\right] \\
& \cdot\left[\begin{array}{ccc}
c_{x x_{\mathrm{A}}} & c_{x y_{\mathrm{A}}} & c_{x z_{\mathrm{A}}} \\
0 & c_{y y_{\mathrm{A}}} & c_{y z_{\mathrm{A}}} \\
0 & 0 & c_{z z_{\mathrm{A}}}
\end{array}\right] \cdot\left[\begin{array}{l}
\theta_{x_{\mathrm{A}}} \\
\theta_{y_{\mathrm{A}}} \\
\theta_{z_{\mathrm{A}}}
\end{array}\right] .
\end{aligned}
$$

For GRACE-FO B, the indices A should be exchanged into B in Eq. (24). Linear coefficients of $c_{x}, c_{y}$, and $c_{z}$ are estimated to be in the order of a few $\mu \mathrm{m} \mathrm{rad}^{-1}$ and quadratic coefficients of $c_{x y}$ and $c_{x z}$ are in the order of a few $\mu \mathrm{m} \mathrm{rad}^{-2}$. Error-free time series of $\theta_{x}, \theta_{y}, \theta_{z}$ were used to simulate $\delta \rho_{\mathrm{ALQ}}$. Figure 16 shows time series of ALQ pointing jitter coupling for 1 month of GRACE-FO A. 

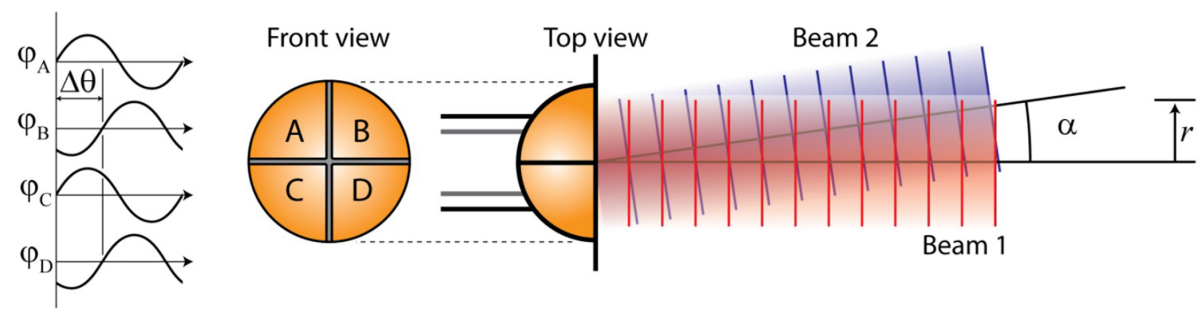

Figure 17. Differential wavefront sensing principle. Two beams of radius $r$ with a relative wavefront tilt of $\alpha$ are detected by a quadrant photodetector. The two beams also have a slight frequency difference (from Sheard et al., 2012).
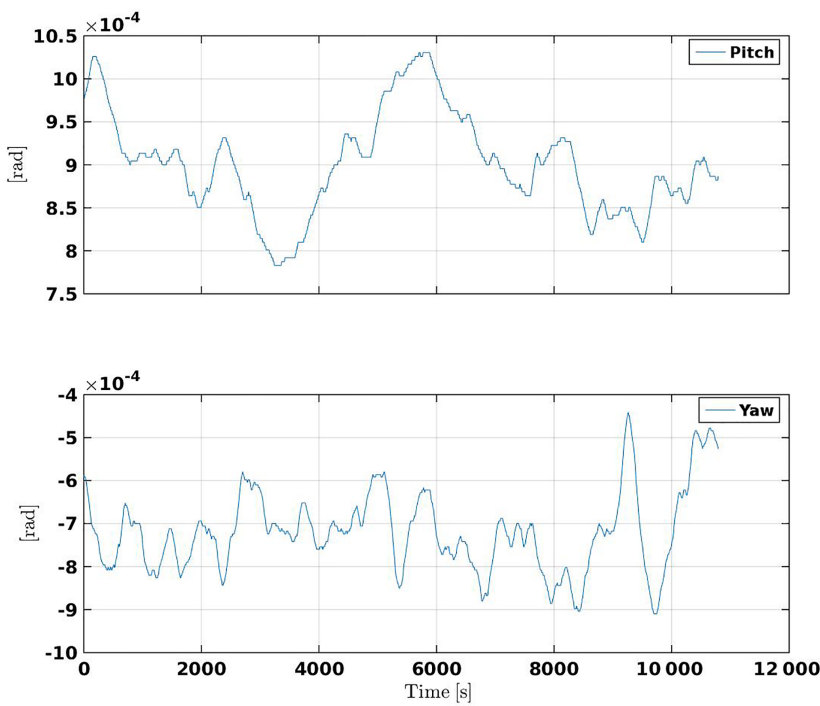

Figure 18. Simulated DWS pitch and yaw angles during two orbital revolutions for GRACE-FO A.

\subsection{Differential wavefront sensing: pitch and yaw measurements}

Differential wavefront sensing (DWS) is a well-known technique for measuring the relative wavefront misalignment between two laser beams with high sensitivity (Sheard et al., 2012). Figure 17 illustrates the basic principle of DWS. DWS provides two extra measurements of the satellite attitude: yaw and pitch pointing angles with respect to the line of sight.

DWS angle measurements on-board GRACE-FO are obtained from the steering mirror on the LRI optical bench (Sheard et al., 2012). The steering mirror orientation is controlled using the DWS error signals constantly driving the error signals back to zero. The steering mirror orientation is recorded as pitch and yaw angles. However, the steering mirror can only turn in discrete units of $4.5 \mu \mathrm{rad}$ around the pitch axis and $6 \mu \mathrm{rad}$ around the yaw axis. Therefore, the angle determination is limited to integer multiples of these units.
For each satellite, DWS pitch and yaw measurements were simulated by

$$
\begin{aligned}
\theta_{y, \text { DWS }} & =\operatorname{round}\left(\frac{\theta_{y}}{4.5 \times 10^{-6} \mathrm{rad}}\right) \cdot 4.5 \times 10^{-6} \mathrm{rad} \\
& +\Delta \theta_{y, \text { DWS }} \\
\theta_{z, \text { DWS }} & =\operatorname{round}\left(\frac{\theta_{z}}{6.0 \times 10^{-6} \mathrm{rad}}\right) \cdot 6.0 \times 10^{-6} \mathrm{rad} \\
& +\Delta \theta_{y, \text { DWS }}
\end{aligned}
$$

where "round" means rounding towards the nearest integer; $\theta_{y}$,DWs and $\theta_{z}$,DWs are the simulated DWS pitch and yaw angles, and $\theta_{y}$ and $\theta_{z}$ are the error-free pitch and yaw angles. The biases $\left(\Delta \theta_{y}\right.$,DWs, $\left.\Delta \theta_{y \text {,DWS }}\right)$ stem mainly from a misalignment of the LRI frame with respect to the SF, which is expected to be within the range of a few milliradians. Figure 18 shows simulated DWS pitch and yaw angles.

\section{Data availability}

The simulated instrument GRACE-FO data are available via https://doi.org/10.22027/AMDC2.

\section{Conclusions}

We have described the simulation of observation and noise models for the GRACE-FO multi-sensor system consisting of inter-satellite ranging with microwave and laser ranging instruments, GPS orbit tracking, accelerometry, and attitude sensing. For the first time, simulated LRI data that include DWS attitude information were generated. The simulated LRI ranging and attitude data may be used in different data analysis scenarios for GRACE-FO, such as the combination of KBR and LRI data and the calibration or estimation of geometric corrections for both KBR and LRI ranging.

On the other hand, different Earth's gravity field solutions derived from actual satellite data can only be compared against each other because the real Earth's gravity field is not known. This is a major problem in the evaluation of the performance of gravity field recovery approaches. A closedloop simulation starting with a known gravity field provides the opportunity to overcome this problem by comparing the 
input gravity field and the gravity field solutions. Also, the effect of instrument noise on gravity field solutions can be investigated by comparing observation residuals with the simulated instrument noise. 


\section{Appendix A}

In the following, we briefly describe the models for atmospheric drag and solar radiation pressure that were used for the orbit simulations.

\section{A1 Atmospheric drag model}

The acceleration due to atmospheric drag is calculated with the following formula from aerodynamic theory (e.g. Montenbruck and Gill, 2000, p. 84), with $A$ as the satellite's crosssectional area, $m$ the mass of the satellite, $\boldsymbol{e}_{\mathrm{v}}$ the unit vector of the velocity relative to the atmosphere, $C_{\mathrm{D}}$ the drag coefficient, and $p$ the atmospheric density at the location of the satellite:

$\ddot{\boldsymbol{r}}=-\frac{1}{2} C_{\mathrm{D}} \frac{A}{m} p v_{r}^{2} \cdot \boldsymbol{e}_{\mathrm{v}}$.

For the calculation of the relative velocity $\boldsymbol{v}_{\mathrm{r}}$, the assumption is made that the atmosphere co-rotates with the Earth. This leads to

$\boldsymbol{v}_{\mathrm{r}}=\dot{\boldsymbol{r}}-\boldsymbol{\omega}_{\oplus} \times \boldsymbol{r}$,

with $\dot{\boldsymbol{r}}$ as the inertial velocity vector of the satellite, $\boldsymbol{r}$ the position vector, and $\boldsymbol{\omega}_{\oplus}$ the Earth's angular velocity; $v_{r}^{2}$ in Eq. (A1) is then the square of the absolute relative velocity:

$v_{\mathrm{r}}^{2}=\left|\boldsymbol{v}_{\mathrm{r}}\right|^{2}$.

\section{A2 Solar radiation pressure model}

A satellite exposed to radiation from the Sun experiences a force arising from the absorption and reflection of incident photons. The resulting acceleration was modelled by using

$$
\begin{aligned}
\ddot{\boldsymbol{r}} & =-v \frac{P_{\odot}}{m}\left(\frac{\mathrm{AU}}{x}\right)^{2} \cdot \sum_{i}\left[\cos \left(\alpha_{i}\right) A_{i} \cdot\left(\left(1-\zeta_{i}\right) \cdot \boldsymbol{e}\right)\right] \\
& +2 \zeta_{i} \cos \left(\alpha_{i}\right) \cdot \boldsymbol{n}_{i} \\
& =-v \frac{P_{\odot}}{m}\left(\frac{\mathrm{AU}}{x}\right)^{2} \cdot \sum_{i}\left[\langle \boldsymbol { n } _ { i } , \boldsymbol { e } \rangle A _ { i } \cdot \left(\left(1-\zeta_{i}\right) \cdot \boldsymbol{e}\right.\right. \\
& \left.\left.+2 \zeta_{i}\left\langle\boldsymbol{n}_{i}, \boldsymbol{e}\right\rangle \cdot \boldsymbol{n}_{i}\right)\right]
\end{aligned}
$$

where the sum is to be taken over all satellite surfaces $i$ that are illuminated by the sunlight, i.e. over all surfaces $i$ such that

$\cos \left(\alpha_{i}\right)=\left\langle\boldsymbol{n}_{i}, \boldsymbol{e}\right\rangle>0$.

Here, $\alpha_{i}$ is the angle of incidence, $\boldsymbol{n}_{i}$ is the outward pointing normal vector to the surface $i$, and $\boldsymbol{e}$ is the normalised vector pointing from the satellite's CoM towards the Sun; $x$ is the Sun-satellite distance and $A_{i}$ is the area of the surface $i$ so that $\cos \left(\alpha_{i}\right) A_{i}$ is its cross-sectional part. The $\zeta_{i}$ represents the reflection coefficients of the respective surfaces combining reflection coefficients for visible and IR light. $P_{\odot}$ denotes the solar radiation pressure at $1 \mathrm{AU}$ (astronomical unit) of distance from the Sun, with a flux (pressure times speed of light) amounting to about $1367 \mathrm{~W} \mathrm{~m}^{-2}$. The left term under the sum in Eq. (A2) accounts for the absorbed photons and the right term accounts for the photon reflections. The shadow function $v$ is a value between 0 (in shadow) and 1 (fully illuminated) calculated using a geometric shadow model with umbra and penumbra cones, ignoring atmosphere and the flattening of the Earth. For more details, see Montenbruck and Gill (2000).

In the above equations, the total mass for each GRACE-FO satellite is $m=655 \mathrm{~kg}$. A GRACE-FO satellite weighs about $180 \mathrm{~kg}$ more than a GRACE satellite due to the additional payload (Gath, 2016).

\section{Appendix B}

There are several possible definitions of the pointing angles. However, if the rotation direction is clear, the methods differ only in the second order; i.e. the differences are in the order of microradians, at most, which can be considered negligible with respect to the measurement uncertainty.

Inter-satellite pointing can be geometrically interpreted as deviations of the SF from the LOSF (Bandikova et al., 2012). Pointing jitter or variations can be expressed as a sequence of rotations about the roll (i.e. $x_{\mathrm{LOSF}}$ ), pitch (i.e. $\boldsymbol{y}_{\mathrm{LOSF}}$ ), and yaw (i.e. $z_{\mathrm{LOSF}}$ ) axes. The roll, pitch, and yaw angles can be derived from the matrix rotating from SF to LOSF (Fig. 1).

The matrix rotating from SF to ICRF is related to the quaternions by using (Wu et al., 2006)

$$
\mathbf{R}_{\mathrm{SF}}^{\mathrm{ICRF}}=\left[\begin{array}{ccc}
q_{0}^{2}+q_{1}^{2}-q_{2}^{2}-q_{3}^{2} & 2\left(q_{1} q_{2}-q_{0} q_{3}\right) & 2\left(q_{1} q_{3}+q_{0} q_{2}\right) \\
2\left(q_{1} q_{2}+q_{0} q_{3}\right) & q_{0}^{2}-q_{1}^{2}+q_{2}^{2}-q_{3}^{2} & 2\left(q_{2} q_{3}-q_{0} q_{1}\right) \\
2\left(q_{1} q_{3}-q_{0} q_{2}\right) & 2\left(q_{2} q_{3}+q_{0} q_{1}\right) & q_{0}^{2}-q_{1}^{2}-q_{2}^{2}+q_{3}^{2}
\end{array}\right] .
$$

Here, $q$ represents the quaternions mentioned in Sect. 4.

The matrix rotating from ICRF to LOSF is derived from the orbital positions:

$$
\mathbf{R}_{\mathrm{ICRF}}^{\mathrm{LOSF}}=\left[\begin{array}{lll}
x_{\mathrm{LOSF}} & \boldsymbol{y}_{\mathrm{LOSF}} & z_{\mathrm{LOSF}}
\end{array}\right],
$$

where the LOSF axes are column vectors, according to the definition in Sect. 2, expressed in inertial frame. Then, the pointing angles (roll $\theta_{x}$, pitch $\theta_{y}$, and yaw $\theta_{z}$ ) can be computed from the rotation matrix $\mathbf{R}_{\mathrm{SF}}^{\mathrm{LOSF}}=\mathbf{R}_{\mathrm{ICRF}}^{\mathrm{LOSF}} \cdot \mathbf{R}_{\mathrm{SF}}^{\mathrm{ICRF}}$ by us- 
ing

$\theta_{x}=\arctan \left(\frac{R_{32}}{R_{33}}\right)$

$\theta_{y}=-\arcsin \left(R_{31}\right)$

$\theta_{z}=\arctan \left(\frac{R_{21}}{R_{11}}\right)$,

where $R_{i j}$ represents the elements of $\mathbf{R}_{\mathrm{SF}}^{\mathrm{LOSF}}$. Here, the first index refers to the row and the second index refers to the column.

\section{Appendix C}

The following is a numerically stable pseudocode to compute quaternions from a given rotation matrix, where $\mathbf{R}$ denotes the rotation matrix and $R(i, j)$ its element in the $i$ th row and $j$ th column.

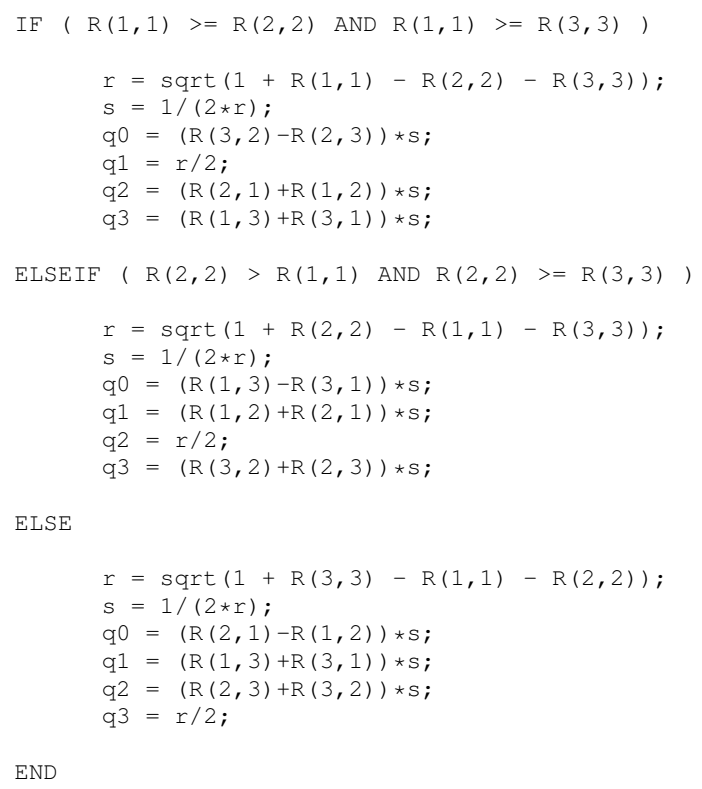

\section{Appendix D}

Tables D1 and D2 describe the format of the data records for KBR1B and LRI1B simulated files. For consistency the tables are similar to Case et al. (2002). 
Table D1. KBR data format record (KBR1B).

\begin{tabular}{llll}
\hline Parameter & Definition & Format & Units \\
\hline gps_time & GPS time, seconds past 12:00:00 noon 1 Jan 2000 & $9 \mathrm{i}$ & $\mathrm{s}$ \\
range & Range between GRACE A and B & $16.10 \mathrm{f}$ & $\mathrm{m}$ \\
range_rate & Range rate between GRACE A and B & $18.16 \mathrm{f}$ & $\mathrm{m} \mathrm{s}$ \\
range_accl & Range acceleration between GRACE A and B & $21.18 \mathrm{f}$ & $\mathrm{m} \mathrm{s}^{-2}$ \\
ioni_corr & Ionospheric range correction between & $16.15 \mathrm{f}$ & $\mathrm{m}$ \\
& GRACE A and B for Ka frequencies & & \\
lighttime_corr & Light time range correction between & $16.15 \mathrm{e}$ & $\mathrm{m}$ \\
& GRACE A and B & & \\
lighttime_rate & Light time range rate correction between & $16.15 \mathrm{e}$ & $\mathrm{m} \mathrm{s}-1$ \\
& GRACE A and B & & \\
lighttime_accl & Light time range acceleration correction between & $16.15 \mathrm{e}$ & $\mathrm{m} \mathrm{s}$ \\
& GRACE A and B & & \\
ant_centr_corr & Antenna phase centre range correction & $16.15 \mathrm{f}$ & $\mathrm{m}$ \\
ant_centr_rate & Antenna phase centre range rate correction & $16.15 \mathrm{e}$ & $\mathrm{m} \mathrm{s}-1$ \\
ant_centr_accl & Antenna phase centre range acceleration correction & $16.15 \mathrm{e}$ & $\mathrm{m} \mathrm{s}-2$ \\
K_A_SNR & SNR K band for GRACE A & $3 \mathrm{i}$ & $0.1 \mathrm{db}-\mathrm{Hz}$ \\
Ka_A_SNR & SNR Ka band for GRACE A & $3 \mathrm{i}$ & $0.1 \mathrm{db}-\mathrm{Hz}$ \\
K_B_SNR & SNR K band for GRACE B & $3 \mathrm{i}$ & $0.1 \mathrm{db}-\mathrm{Hz}$ \\
Ka_B_SNR & SNR Ka band for GRACE B & $3 \mathrm{i}$ & $0.1 \mathrm{db}-\mathrm{Hz}$ \\
qualfig & 0 = not defined & $0.8 \mathrm{i}$ & $\mathrm{n} / \mathrm{a}$ \\
\hline
\end{tabular}

n/a: not applicable

Table D2. LRI data format record (LRI1B).

\begin{tabular}{llll}
\hline Parameter & Definition & Format & Units \\
\hline gps_time & GPS time, seconds past 12:00:00 noon 1 Jan 2000 & $9 \mathrm{i}$ & $\mathrm{s}$ \\
range & Range between GRACE A and B & $19.10 \mathrm{f}$ & $\mathrm{m}$ \\
range_rate & Range rate between GRACE A and B & $19.16 \mathrm{f}$ & $\mathrm{m} \mathrm{s}^{-1}$ \\
range_accl & Range acceleration between GRACE A and B & $22.19 \mathrm{f}$ & $\mathrm{m} \mathrm{s}^{-2}$ \\
lighttime_corr & Light time range correction between & $16.15 \mathrm{e}$ & $\mathrm{m}$ \\
& GRACE A and B & & \\
lighttime_rate & Light time range rate correction between & $16.15 \mathrm{e}$ & $\mathrm{m} \mathrm{s}-1$ \\
& GRACE A and B & $16.15 \mathrm{e}$ & $\mathrm{m} \mathrm{s}-2$ \\
lighttime_accl & Light time range acceleration correction between & $16.15 \mathrm{f}$ & $\mathrm{n} / \mathrm{a}$ \\
& GRACE A and B & $16.15 \mathrm{e}$ & $\mathrm{m} \mathrm{s}-1$ \\
ver_point_corr & Vertex point range correction & $16.15 \mathrm{e}$ & $\mathrm{m} \mathrm{s}-2$ \\
ver_point_rate & Vertex point range rate correction & $19.17 \mathrm{f}$ & $\mathrm{rad}$ \\
ver_point_accl & Vertex point range acceleration correction & $19.17 \mathrm{f}$ & $\mathrm{rad}$ \\
pitch_A_dws & Pitch angle from differential wavefront sensing for GRACE A \\
yaw_A_dws & Yaw angle from differential wavefront sensing for GRACE A & $19.17 \mathrm{f}$ & $\mathrm{rad}$ \\
pitch_B_dws & Pitch angle from differential wavefront sensing for GRACE B & $19.17 \mathrm{f}$ & $\mathrm{rad}$ \\
yaw_B_dws & Yaw angle from differential wavefront sensing for GRACE B & $19 \mathrm{i}$ & $0.1 \mathrm{db}-\mathrm{Hz}$ \\
LRI_A_SNR & SNR LRI for GRACE A & $3 \mathrm{i}$ & $0.1 \mathrm{db}-\mathrm{Hz}$ \\
LRI_B_SNR & SNR LRI for GRACE B & $0.8 \mathrm{i}$ & $\mathrm{n} / \mathrm{a}$ \\
qualflg & 0= not defined & & \\
\hline
\end{tabular}


Author contributions. MN developed the orbit integrator code and performed the orbit simulations. HW and VM developed the instrument noise models. GH and MH developed the LRI noise models. ND performed the instrument noise simulations and prepared the paper with contributions from all co-authors.

Competing interests. The authors declare that they have no conflict of interest.

Acknowledgements. This project is supported by funding from the SFB 1128 "Relativistic Geodesy and Gravimetry with Quantum Sensors (geo-Q)" by the Deutsche Forschungsgemeinschaft. We also thank JPL and AIRBUS Defense and Space for providing the GRACE-FO attitude and orbital control system performance predictions.

Edited by: David Carlson

Reviewed by: two anonymous referees

\section{References}

Bandikova, T., Flury, J., and Ko, U.: Characteristics and accuracies of the GRACE inter-satellite pointing, Adv. Space Res., 50, 123135, 2012.

Case, K., Kruizinga, G., and Wu, S.: GRACE level 1B data product user handbook, JPL Publication D-22027, 2002.

Flechtner, F., Neumayer, K.-H., Dahle, C., Dobslaw, H., Fagiolini, E., Raimondo, J.-C., and Güntner, A.: What Can be Expected from the GRACE-FO Laser Ranging Interferometer for Earth Science Applications?, Surv. Geophys., 37, 453-470, https://doi.org/10.1007/s10712-015-9338-y, 2016.

Franklin, J. N.: Numerical simulation of stationary and nonstationary gaussian random processes, SIAM Review, 7, 68-80, 1965.

Gath, P.: Integration und Test der GRACE Follow-On Satelliten, Tech. Rep. 420305, Deutscher Luft- und Raumfahrtkongress, 2016.

Gerlach, C., Földvary, L., Švehla, D., Gruber, T., Wermuth, M., Sneeuw, N., Frommknecht, B., Oberndorfer, H., Peters, T., Rothacher, M., Rummel, R., and Steigenberger, P.: A CHAMP-only gravity field model from kinematic orbits using the energy integral, Geophys. Res. Lett., 30, 2037, https://doi.org/10.1029/2003GL018025, 2003.

Harvey, N.: GRACE star camera noise, Adv. Space Res., 58, 408414, 2016

Horwath, M., Lemoine, J., Biancale, R., and Bourgogne, S.: Improved GRACE science results after adjustment of geometric biases in the Level-1B K-band ranging data, J. Geodesy, 85, 23-38, 2011.
Kim, J.: Simulation study of a low-low satellite-to-satellite tracking mission, PhD thesis, The University of Texas at Austin, 2000.

Mayer-Gürr, T.: Gravitationsfeldbestimmung aus der Analyse kurzer Bahnbögen am Beispiel der Satellitenmissionen CHAMP und GRACE, Ph.D. thesis, Universitäts-und Landesbibliothek Bonn, 2006.

Montenbruck, O. and Gill, E.: Satellite Orbits, Springer-Verlag, Berlin Heidelberg, 2000.

Müller, V.: Orbit simulation Toolkit, Ph.D. thesis, Bachelor of Science Degree Thesis, Max-Planck-Institute for Gravitational Physics (Albert Einstein Institute, AEI) and Gottfried Wilhelm Leibniz Universtitut, Hannover, 2010.

Petit, G. and Luzum, B.: IERS Conventions (2010), Tech. Rep. IERS Technical Note No. 36, Frankfurt am Main: Verlag des Bundesamts für Kartographie und Geodäsie, 2010.

Reigber, C.: Gravity field recovery from satellite tracking data, in: Theory of satellite geodesy and gravity field determination, 197234, Springer, 1989.

Rieser, D., Mayer-Gürr, T., Savcenko, R., Bosch, W., Wünsch, J., Dahle, C., and Flechtner, F.: The ocean tide model EOT11a in spherical harmonics representation, Technical Note, Institute of Theoretical Geodesy and Satellite Geodesy (ITSG), TU Graz, Austria, 2012.

Rummel, R.: Determination of short-wavelength components of the gravity field from satellite-to-satellite tracking or satellite gradiometry-an attempt to an identification of problem areas, Manuscripta Geodaetica, 4, 107-148, 1979.

Sheard, B., Heinzel, G., Danzmann, K., Shaddock, A., Klipstein, W., and Folkner, W.: Intersatellite laser ranging instrument for the GRACE follow-on mission, J. Geod., 86, 1083-1095, 2012.

Standish, E. M.: JPL Planetary and Lunar Ephemerides, DE405/LE405, Jet Propulsion Laboratory Interoffice Memorandum, IOM 312.F - 98 - 048, 1998.

Stanton, R., Bettadpur, S., Dunn, C., Renner, K.-P., and Watkins, M.: Gravity Recovery And Climate Experiment (GRACE) science \& mission requirements document, GRACE 327-200, Jet Propulsion Laboratory, California Institute of Technology, 1998.

Tapley, B. D., Bettadpur, S., Watkins, M., and Reigber, C.: The gravity recovery and climate experiment: Mission overview and early results, Geophys. Res. Lett., 31, L09607, https://doi.org/10.1029/2004GL019920, 2004.

Wahr, J., Molenaar, M., and Bryan, F.: Time variability of the Earth's gravity field: Hydrological and oceanic effects and their possible detection using GRACE, J. Geophys. Res.-Solid Earth 103, 30205-30229, https://doi.org/10.1029/98JB02844, 1998.

Watkins, M., Flechtner, F., Webb, F., Landerer, F., and Grunwald, L.: Current Status of the GRACE Follow-On Mission, Presented at the European Geosciences Union General Assembly, Vienna, Austria, 2016.

Wu, S.-C., Kruizinga, G., and Bertiger, W.: Algorithm theoretical basis document for GRACE level-1B data processing V1.2, GRACE 327-741, Jet Propulsion Laboratory, California Institute of Technology, 2006. 